\title{
A simple mathematical standardized measurement of acetabulum anteversion after total hip arthroplasty
}

\author{
Chen-Kun Liaw ${ }^{\mathrm{a}, \mathrm{d} 1,5}$, Rong-Sen Yang ${ }^{\mathrm{b} 2,5}$, Sheng-Mou Hou ${ }^{\mathrm{b} 3}$, Tai-Yin $\mathrm{Wu}^{\mathrm{c} 4}$ \\ and Chiou-Shann Fuh ${ }^{\mathrm{d} *}$ \\ ${ }^{a}$ Department of Orthopaedics, Tao-Yuan General Hospital, Taiwan; ${ }^{b}$ Department of Orthopaedics, \\ College of Medicine, National Taiwan University and Hospital, Taiwan; ${ }^{c}$ Taipei City Hospital, \\ Renai Branch, Taipei, Taiwan; ${ }^{d}$ Institute of Computer Science and Information Engineering, \\ National Taiwan University, Taipei, Taiwan
}

(Received 27 August 2007; final version received 12 December 2007)

\begin{abstract}
We invented a standardization method to measure the cup's anteversion after total hip arthroplasty without the influence of patient's position. We measured 68 radiographs of 10 patients after total hip replacement (THR) and calculated the error of each measurement, defined as the difference with the average of the same measuring method on the same patient. We also calculated the repeatability standard deviation (RSD) of each method according to the American Society for Testing and Materials, ASTM E691.

Mean absolute inter-examination angle error, defined as the average of the absolute deviations, was $0.75^{\circ}$ for standardized anteversion (range $0.03-2.51^{\circ}$ ), as compared with those without standardization, $2.30^{\circ}$ (range $0.04-13.04^{\circ}$ ). The inter-examination measurement reliability (precision), defined as one RSD, was $0.99^{\circ}$ for standardized anteversion, as compared with those without standarization, $3.50^{\circ}$. There is no difference between patients four and five without $(p=0.097)$. There is a significant difference with standardization $(p<0.0001)$.

Our study demonstrated that this mathematical method is a precise tool to measure the anteversion of the acetabular cup. We hope that it can be used widely in the future.
\end{abstract}

Keywords: acetabulum; anteversion; standardized; radiographs

Mathematics Subject Classification: 92b05; 92 b99

\section{Introduction}

The anteversion of acetabulum cup determines the range of motion, stability as well as the function after total hip arthroplasty. Many methods have been proposed for the measurement of anteversion on the plain radiograms $[1,4-8,10,13,14]$. However, the radiographs are all taken by different technicians at different times. The major difference of radiographic anteversion is related to the different patient positioning [11]. Most of the methods did not correct it. Therefore, we need a standardization process to clarify the influence of patient positioning and also to differentiate the real cup movement shown in anteversion angle between serial measurements. A computer tomography (CT) method has been proposed for standardization with a good inter- and intra-observer reliability [9]. Such a method adopted an anterior pelvic plane by $\mathrm{CT}$ images for measurement. In addition to high dose radiation and medical cost, it may be neither suitable nor practical if the CT

*Corresponding author. Email: d92008@csie.ntu.edu.tw 
images were not available in the clinical practice, especially for a serial measurement. Therefore, it is more practical to develop a standardization method for measurement of the anteversion angle on plain radiograms.

In this study, we propose a method standardized by mathematical vector equation and compare the anteversion angles measured with or without standardization on 68 radiographs of 10 patients after total hip replacement.

\section{Materials and methods}

To standardize the plain radiographs, we first define the pelvis radiographic axis as the axis from centre of sacrococcygeal junction pointed toward the upper pole of symphysis pubis. The upper pole of symphysis pubis means the mid point of the upper end of interpubic fibrocartilage. We define the radiographic standardized anteversion (also called Liaw's version) as the angle between the plane of acetabulum and pelvis radiographic axis (Figure 1).

With this definition of standardized anteversion, we can measure it when the X-ray beam is just parallel to the pelvis radiographic axis. Unfortunately, most radiographs do not meet this requirement. We can solve this problem in two ways. First, we can adjust the patient's and the tube's positions, again and again, until the X-ray beam is just parallel to this axis and centred at the hip. This process is not practical as patients may have dozens of radiographs without any well qualified ones. Second, we can correct the

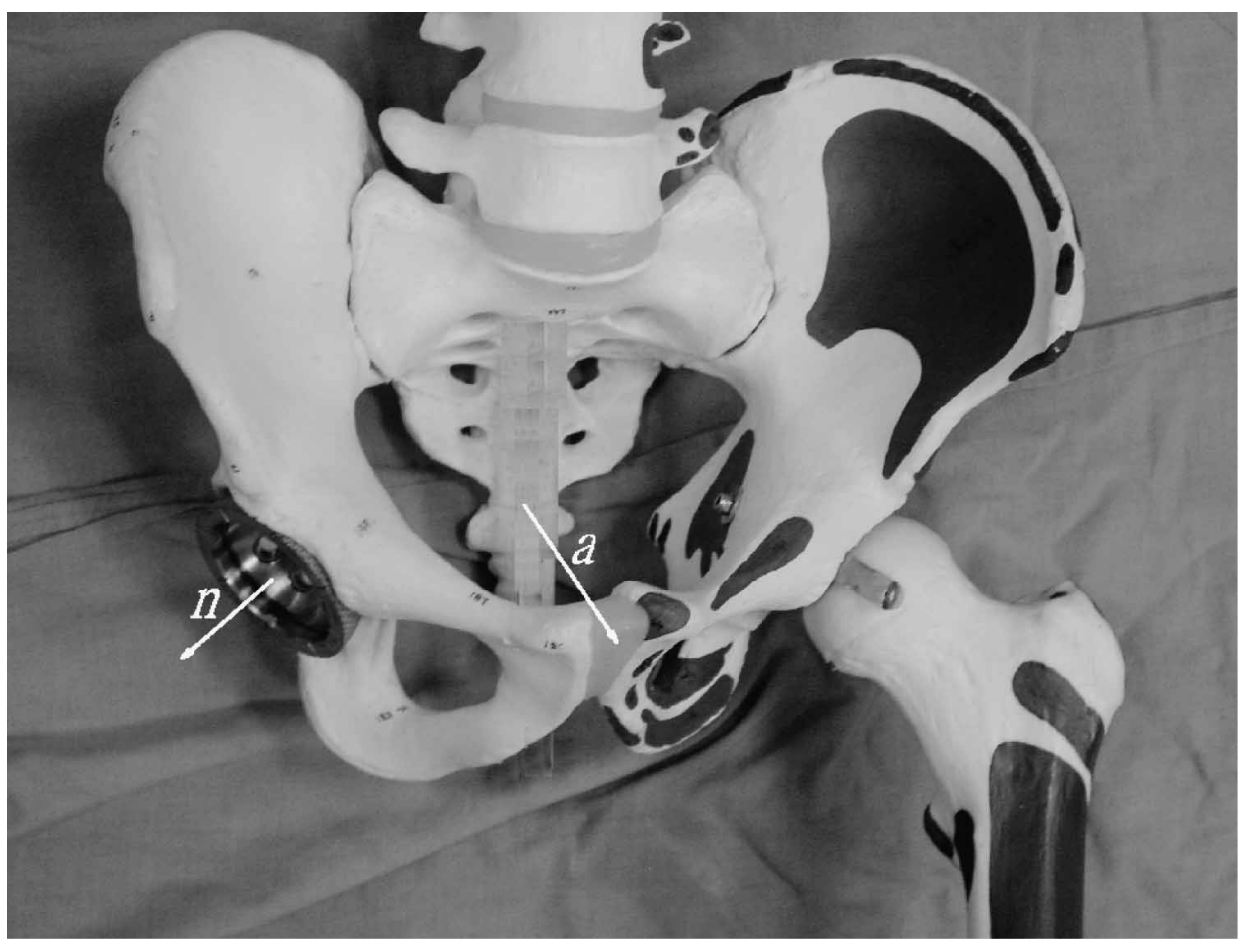

Figure 1. Vector $a$ represents pelvis radiographic axis, which is defined as axis from centre of sacrococcygeal junction pointed toward the upper pole of symphysis pubis. The acetabulum cup is placed on the right hip. Vector $n$ represents the normal vector of the acetabulum. 
measured anteversion by standardization process with mathematical methods. Through the plain radiographs, we can find some clues for standardization.

Radiographic standardized anteversion $=$

$$
\arcsin \left(\frac{-h \times \sin \varphi \times \cos \theta+v \times \cos \varphi \times \cos \theta+\sin \theta \times\left(\operatorname{ssd}^{2}-h^{2}-v^{2}\right)^{0.5}}{\operatorname{ssd}}\right)
$$

The 'arc sin' function may return radian value. We can convert it to degrees by multiplying 180/3.1416.

Equation (1) is our standardizing equation, where the ssd in mm means the length from upper pole of symphysis pubis to sacrococcygeal junction, and where the $h$ in mm means the horizontal displacement of sacrococcygeal junction related to the upper pole of symphysis pubis in horizontal direction. We assign $h$ to be positive if sacrococcygeal junction is between this acetabulum and the upper pole of symphysis pubis, otherwise it is negative. The $v$ in mm means vertical displacement of sacrococcygeal junction related to the upper pole of symphysis pubis in a vertical direction. We assign $v$ to be positive if sacrococcygeal junction is above the upper pole of symphysis pubis, otherwise it is negative (Figure 2). The $\theta$ means the radiographic (planar) anteversion angle. The $\varphi$ means the inclination (abduction) angle.

The detailed deduction process is shown in Appendix A.

In this equation, ssd is unknown. We measured ssd on 109 radiographs from crosstable lateral view from 109 adult patients ( $>18$ years old), including 51 females and 58 males. Considering when taking radiographs, the distance between the ssd and radiograph sensor is almost twice as long as the acetabulum in antero-posterior view, the results of ssd should be multiplied by 0.9 to approximate the real situation. The result is adopted for calculation in the equation.

Once the method is established, we need to verify our method. First, we collect the initial 11 patients who received total hip arthroplasty in our hospital in 1999. The inclusion criterion for radiographs is that the radiographs must include the acetabulum, symphis pubis, and sacrococcygeal junction. Those who averaged radiographic anteversion less than $10^{\circ}$ are excluded, because anteversion and retroversion are difficult to differentiate in this situation. Furthermore, we do not compare the difference of inclination, because Tannast et al. [11] reported that pelvis orientation has little influence on the inclination. The tilt of the X-ray films was also not taken into consideration in this study.

In total, 10 patients and 68 radiographs are included; two are femoral antero-posterior radiographs, three are kidney, ureters and urinary bladder radiographs, five are hip anteroposterior radiographs, and 58 are pelvis antero-posterior radiographs. We measured the radiographic anteversion and inclination by the method of Lewinnek et al. [6], and then radiographic standardized anteversion by our method. All these measurements were done by the same author, and he did not know the result standardization until he had finished all the measurements to eliminate bias from prior data.

We also calculated the difference between patients \#4 and \#5 before and after standardization.

\section{Statistical methods}

We calculated the mean and standard deviation of the ssd, and used a $t$-test to check the effect of gender-related difference. We calculated the standard deviation for all 


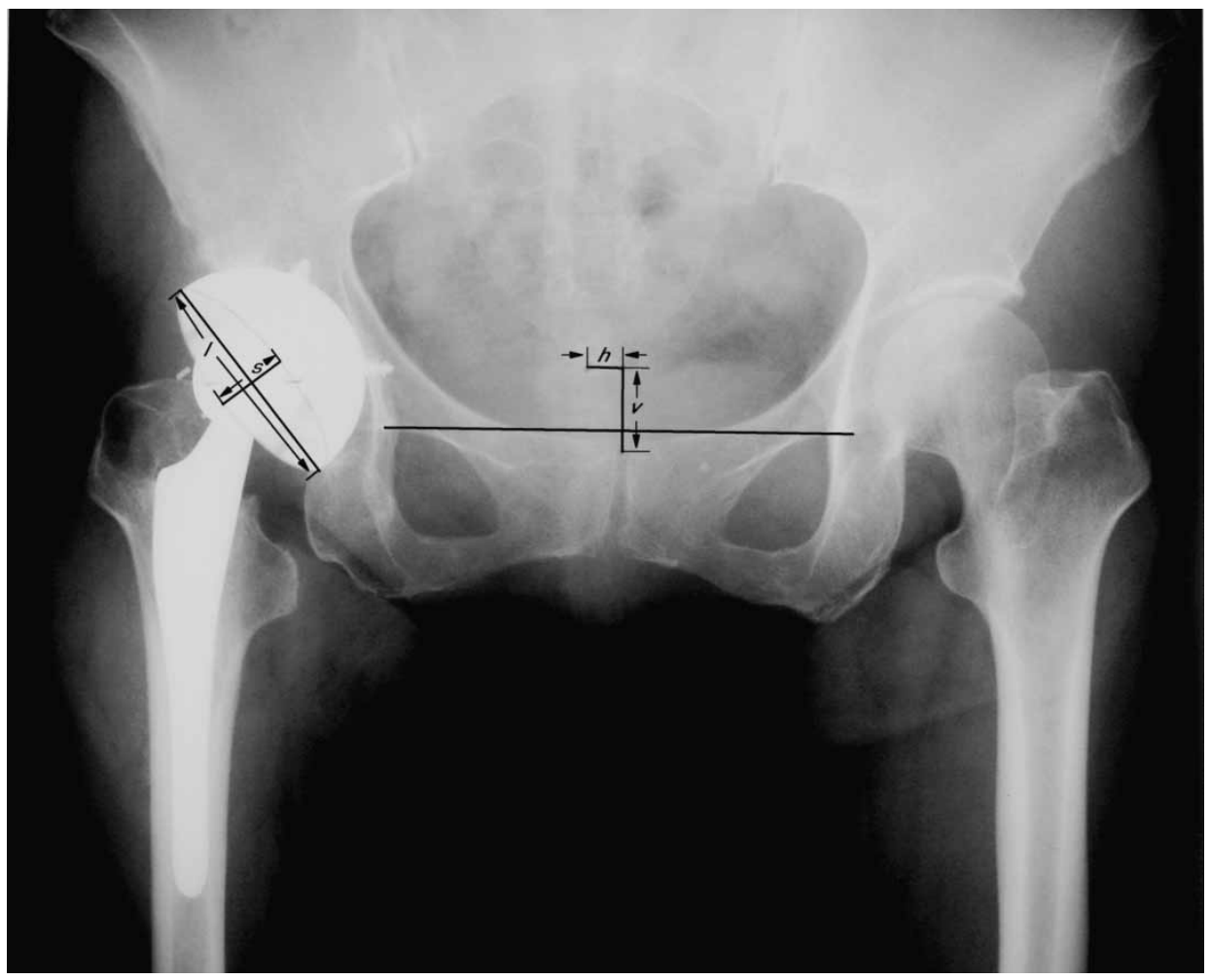

Figure 2a. Radiograph of a patient who underwent right total hip arthroplasty. We measured the version of acetabulum by Lewinnek's method (anteversion $=\operatorname{arc} \sin (s / l), s$ means short axis of the ellipse, $l$ means long axis of the ellipse), and inclination. In this case, the radiographic version was 18.1 degrees and inclination was 48 degrees. We measured vertical displacement $v$ as displacement of sacrococcygeal junction related to the upper pole of symphysis pubis in $Y$-axis direction. We assign $v$ to be positive if sacrococcygeal junction is above the upper pole of symphysis pubis. In this case, the vertical displacement $v$ is positive. We measured horizontal displacement $h$ as displacement of sacrococcygeal junction related to the upper pole of symphysis pubis in $X$-axis direction. In this case, the displacement $h$ was positive because the sacrococcygeal junction is between this acetabulum and the upper pole of symphysis pubis. In this case, $h$ was $10 \mathrm{~mm}$ and $v$ was $25 \mathrm{~mm}$. By Equation (1), we calculated a standardized version. In this case, if we assigned $\theta$ to be positive (anteversion) then the standardized anteversion was 21.4 degrees. If we assigned $\theta$ to be negative (retroversion) the standardized anteversion became $-14.3^{\circ}$.

measurements of each patient and repeatability standard deviation (RSD) from ASTM E691 for comparison [2].

$$
s=\sqrt{\frac{\sum_{j=1}^{c} \sum_{i=1}^{n}\left(X_{i j}-\bar{X}_{j}\right)^{2}}{\sum_{j=1}^{c}\left(n_{j}-1\right)}}
$$

where $S$ means RSD. $X_{i j}$ means the result of the $j$ th patient, the $i$ th X-rays' measurement. $\bar{X}_{j}$ means the mean result of the $j$ th patient and $n_{j}$ means the total number of measurements of the $j$ th patient.

Small RSD means a good repeatability of the measurement method.

To compare patients four and five, we used a $t$-test. The probability of less than 0.01 is significantly different. 


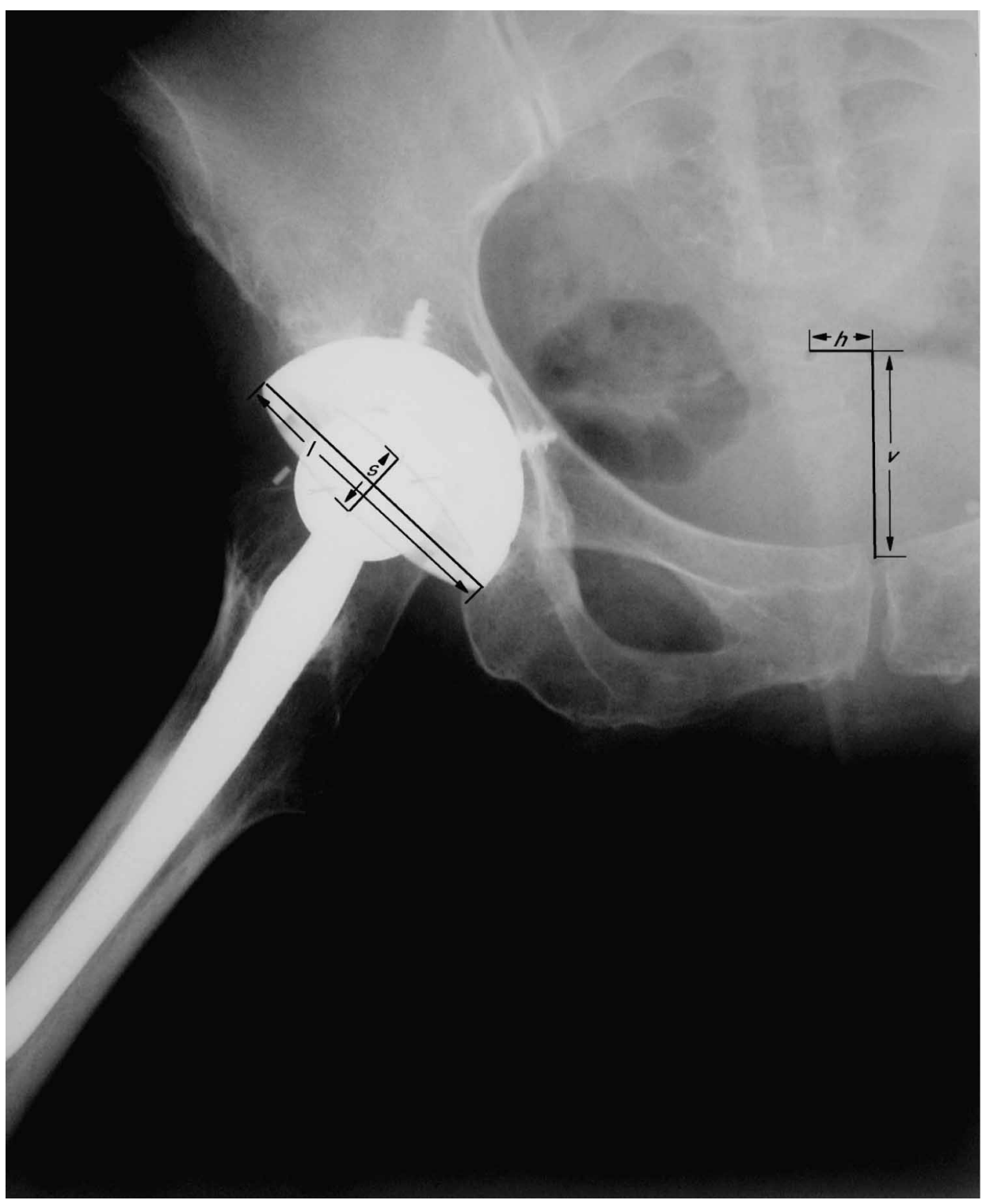

Figure $2 \mathrm{~b}$. The hip antero-posterior radiograph with the same patient was shown. The radiographic version was 14.1 degrees. The inclination was 48 degrees. In this case, $h$ was $14 \mathrm{~mm}$ and $v$ was $47 \mathrm{~mm}$. If we assigned $\theta$ to be positive (anteversion) then the standardized anteversion was 21.8 degrees. If we assign $\theta$ to be negative (retroversion) the standardized anteversion became -5.0 degrees. Comparing with Figure 2a's result, we confirmed it to be anteversion, because the difference of $0.4(=21.8-21.4)$ degrees was much smaller than $9.3(=14.3-5.0)$ degrees.

\section{Results}

The body height ranged from $142-179 \mathrm{~cm}$ with a mean of $162 \mathrm{~cm}$ for these subjects, aged 20-92 years (mean 51 years). For male patients, the ssd ranged from 137 to $190 \mathrm{~mm}$ $(\mathrm{mean} \pm \mathrm{SD}=159 \pm 12 \mathrm{~mm})$, and $138-183 \mathrm{~mm}($ mean $\pm \mathrm{SD}=160 \pm 12 \mathrm{~mm})$ for female patients. There is no statistical difference between the two groups $(p=0.91)$. For all 
109 patients, the ssd ranged from 137 to $190 \mathrm{~mm}$ (mean $\pm \mathrm{SD}=159 \pm 12 \mathrm{~mm}$ ). We applied the result 'ssd $=159 \times 0.9=143$ ' to Equation (1) for further calculation.

The results are shown in Table 1 and Figure 3. Mean absolute inter-examination angle error, which is defined as the average of the absolute deviations (MAD), was $0.73^{\circ}$ for the standardized anteversion (range, $0.03-2.51^{\circ}$ ), as compared with the error for those without standarization, $2.30^{\circ}$ (range, $0.04-13.04^{\circ}$ ). The RSD of radiographic anteversion was $3.50^{\circ}$, and for radiographic standardized anteversion, $0.99^{\circ}$. The standard deviations of radiographic anteversion for these 10 patients ranged from 1.11 to $8.73^{\circ}$, and for radiographic standardized anteversion, $0.50-1.90^{\circ}$. The vertical displacement $v$ ranged from -25 to $57 \mathrm{~mm}$ (mean $\pm \mathrm{SD}=29 \pm 18 \mathrm{~mm}$ ). The horizontal displacement $h$ ranged from -29 to $30 \mathrm{~mm}$ (mean $\pm \mathrm{SD}=3 \pm 12 \mathrm{~mm}$ ).

There is no difference between patients \#4 and \#5 with radiographic anteversion $(p=0.097)$. However, after standardization, there is significant difference $(p<0.0001)$.

\section{Discussion}

Currently, there is no plain radiographic method to standardize anteversion. Our method is the first proposed 2D method of standardization. The result shows that the intra-observer reliability is better than that without standardization. In this study, we only measure radiographic anteversion using the method of Lewinnek et al. [6], and then proceed to standardization. We can easily extend the usage to any other method which can measure radiographic anteversion $[1,4-8,10,13,14]$.

In Table 1, our result shows that without standardization, the maximal standard deviation of radiographic anteversion is $8.73^{\circ}$ and MAD, $6.52^{\circ}$. This occurs in patient three. This is because one of the pelvis antero-posterior radiograph was taken with large horizontal deviation $(-29 \mathrm{~mm})$ in comparison with the other three radiographs (16-22 mm). Our standardization method effectively corrected it and got a small standard deviation $\left(1.9^{\circ}\right)$ and MAD $\left(1.46^{\circ}\right)$. This example showed the great correction capability for the bias using our method.

Olivecrona et al. [9] had published the only one standardized method using CT and special software. They used one standard deviation as an estimated operational error, which was between 2.3 and $2.9^{\circ}$. Ours is 0.50 and $1.90^{\circ}$, much more precise than the results using their method. Our 2D method is better than their $3 \mathrm{D}$ method, which is possibly due to the quantization error. The resolution (total number of pixels per inch) for CT is much lower than plain radiographs. Another reason may be the operational error. Our method needs two measurements in the standardization process, while their method had to point out four points.

Tannast et al. [12] reported a way to estimate pelvic tilt and rotation in a similar way. One may estimate standardized version by looking up the graph in Murray's report [8], after having noted tilt and rotation angles. They estimated tilt and rotation by linear regression. The precision is good when the distance is small. However, when the displacement is larger $(>4 \mathrm{~cm})$, the curve is outside the linear portion and the precision decreased. In our experience, this situation happens often, 20 of 68 vertical displacements were larger than $4 \mathrm{~cm}$. It implied the possible limitation of its application. Our method uses precise mathematical equations and the precision is good in all ranges of the displacement.

We did not do an inter-observer study of our standardization method. The equation itself will not cause inter-observer difference and the difference comes from measurement. Our standardization method needs to measure the horizontal and vertical displacements of sacrococcygeal junction related to the upper pole of symphysis pubis. These two measurements are major causes of inter-observer difference. However, Tannast et al. [12] 
Table 1. The results of measured anteversion.

\begin{tabular}{|c|c|c|c|c|c|c|c|c|c|c|c|c|c|}
\hline \multirow[b]{2}{*}{ Patient } & \multirow[b]{2}{*}{$\begin{array}{l}\text { Number of radiograms of } \\
\text { each patient }\end{array}$} & \multicolumn{6}{|c|}{ Radiographic Anteversion } & \multicolumn{6}{|c|}{ Standardized Anteversion } \\
\hline & & Min* & Median & Max* & Mean & STD ${ }^{*}$ & MAD* & Min* & Median & Max* & Mean & STD $^{*}$ & MAD* \\
\hline 1 & 3 & 6.2 & 11.3 & 11.5 & 9.67 & 3.02 & 2.32 & 15.8 & 17.0 & 17.1 & 16.6 & 0.73 & 0.56 \\
\hline 2 & 6 & 7.0 & 12.2 & 18.2 & 12.3 & 3.62 & 2.40 & 17.0 & 18.0 & 18.4 & 17.8 & 0.55 & 0.43 \\
\hline 3 & 4 & 0 & 17.1 & 17.9 & 13.0 & 8.73 & 6.52 & 13.7 & 16.6 & 17.9 & 16.2 & 1.90 & 1.46 \\
\hline 4 & 10 & 10.3 & 13.5 & 21.0 & 13.9 & 3.17 & 2.30 & 27.6 & 30.1 & 31.5 & 29.8 & 1.39 & 1.14 \\
\hline 5 & 11 & 12.9 & 15.3 & 23.1 & 16.4 & 3.43 & 2.59 & 21.1 & 22.4 & 24.1 & 22.6 & 0.99 & 0.81 \\
\hline 6 & 5 & 24.9 & 26.4 & 32.9 & 27.6 & 3.25 & 2.42 & 27.6 & 28.5 & 28.9 & 28.3 & 0.54 & 0.44 \\
\hline 7 & 8 & 11.6 & 13.2 & 14.8 & 13.1 & 1.11 & 0.89 & 23.6 & 24.5 & 25.6 & 24.6 & 0.77 & 0.65 \\
\hline 8 & 5 & 10.8 & 11.4 & 15.1 & 12.3 & 1.73 & 1.33 & 17.0 & 17.4 & 18.3 & 17.6 & 0.56 & 0.47 \\
\hline 9 & 8 & 8.3 & 12.4 & 20.9 & 13.4 & 3.99 & 3.09 & 7.5 & 8.7 & 10.2 & 8.9 & 1.04 & 0.89 \\
\hline 10 & 8 & 9.1 & 12.7 & 14.1 & 12.4 & 1.48 & 0.90 & 14.6 & 15.2 & 16.1 & 15.3 & 0.50 & 0.42 \\
\hline Total: & 68 & & & & & $\begin{array}{l}* * \text { RSD: } \\
3.50\end{array}$ & $\begin{array}{l}\text { Average: } \\
2.30\end{array}$ & & & & & $\begin{array}{c}* * \text { RSD: } \\
0.99\end{array}$ & $\begin{array}{c}\text { Average: } \\
0.75\end{array}$ \\
\hline
\end{tabular}




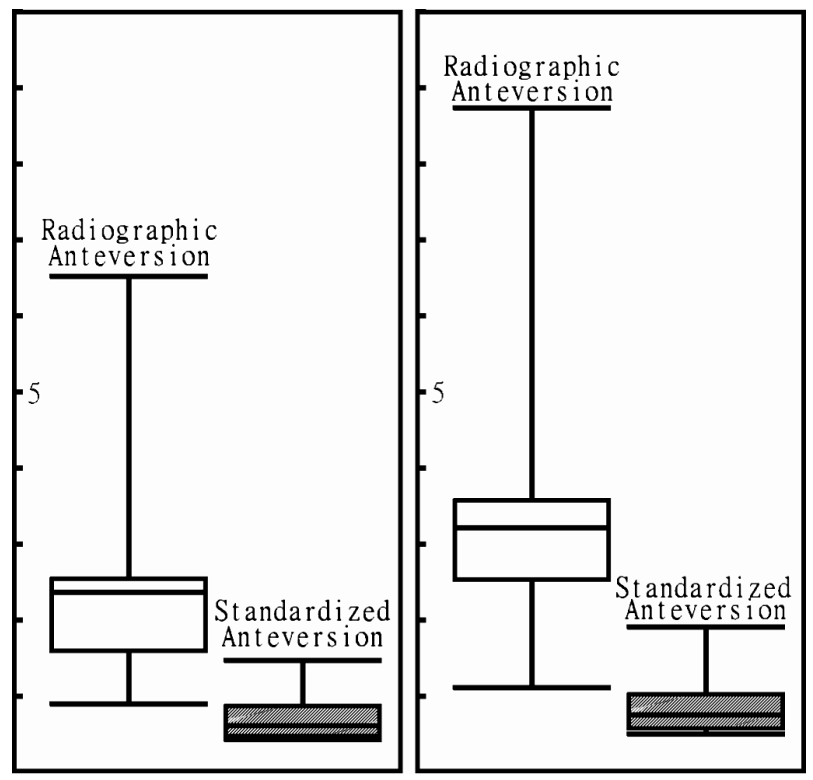

Figure 3. The box plots the absolute deviation (left) and standard deviation (right). It showed that without standardization (radiographic anteversion), the standard and absolute deviation were larger in comparison with the measurement using the standardized method (standardized anteversion).

reported that the inter- and intra- observer correlation of measuring the two distances is good, larger than 0.91 . We can deduce that our inter- and intra- observer correlation should be good too.

Our result showed great progress in precision. Some orthopaedic surgeons may question the necessity of precision however, we think precision is necessary. When comparing two radiographs from the same patient with hip pain, a greater precision can help us distinguish the cup's movement if the anteversion change is small. Any improvement on precision is meaningful. Our results show that without standardization, there is no statistical difference between patients four and five, however, after standardization, there is statistical difference. Lets assume the two patients are one patient with cup movement, for example patient four is before movement while patient five is after movement. Without standardization, we cannot find the movement. However, after standardization, we can easily distinguish it.

We neglected the tilt of X-ray film in this study. We corrected X-ray tube position but not the X-ray film, because we cannot find any clues in it. Fortunately, it does not cause a big error in our result. Our method also neglected the X-ray tube position from the centre in the upper pole of symphysis pubis to centred in the hip. This produces an error of about $3-5$ degrees. The error is the same for each patient. We can easily correct it by measuring distance $d$ from the upper pole of symphysis pubis to the acetabulum centre in centimetres.

$$
\begin{aligned}
& \text { Oblique-projection-corrected-anteversion } \\
& =\theta_{\mathrm{sc}} \operatorname{arc} \sin \left(\frac{100 \times \sin \theta_{s}-d \times \sin \varphi \times \cos \theta_{s}}{100^{2}+d^{2}}\right)^{0.5}
\end{aligned}
$$

The detailed deduction process is shown in Appendix B. 
This can cause additional operational errors, but this correction is the same for each patient.

Another interesting issue is to compare our method with the method of Olivecrona et al. [9]. Regarding the 3D relationship between the pelvis radiographic axis and anterior pelvic plane, we can obtain this relationship by cross-table lateral view. Eckman et al. [3] have proved that we can obtain pelvis flexion from cross-table lateral view. With the same principle, we can obtain the angle between anterior pelvic plane and pelvis radiographic axis from this view by calculating the average of the two angles formed by the two anterior superior iliac spines (ASIS), the pubic tubercle, and the sacrococcygeal junction (Figure 4). According to Appendix C, we can calculate the standardized anteversion referenced by the anterior pelvic plane.

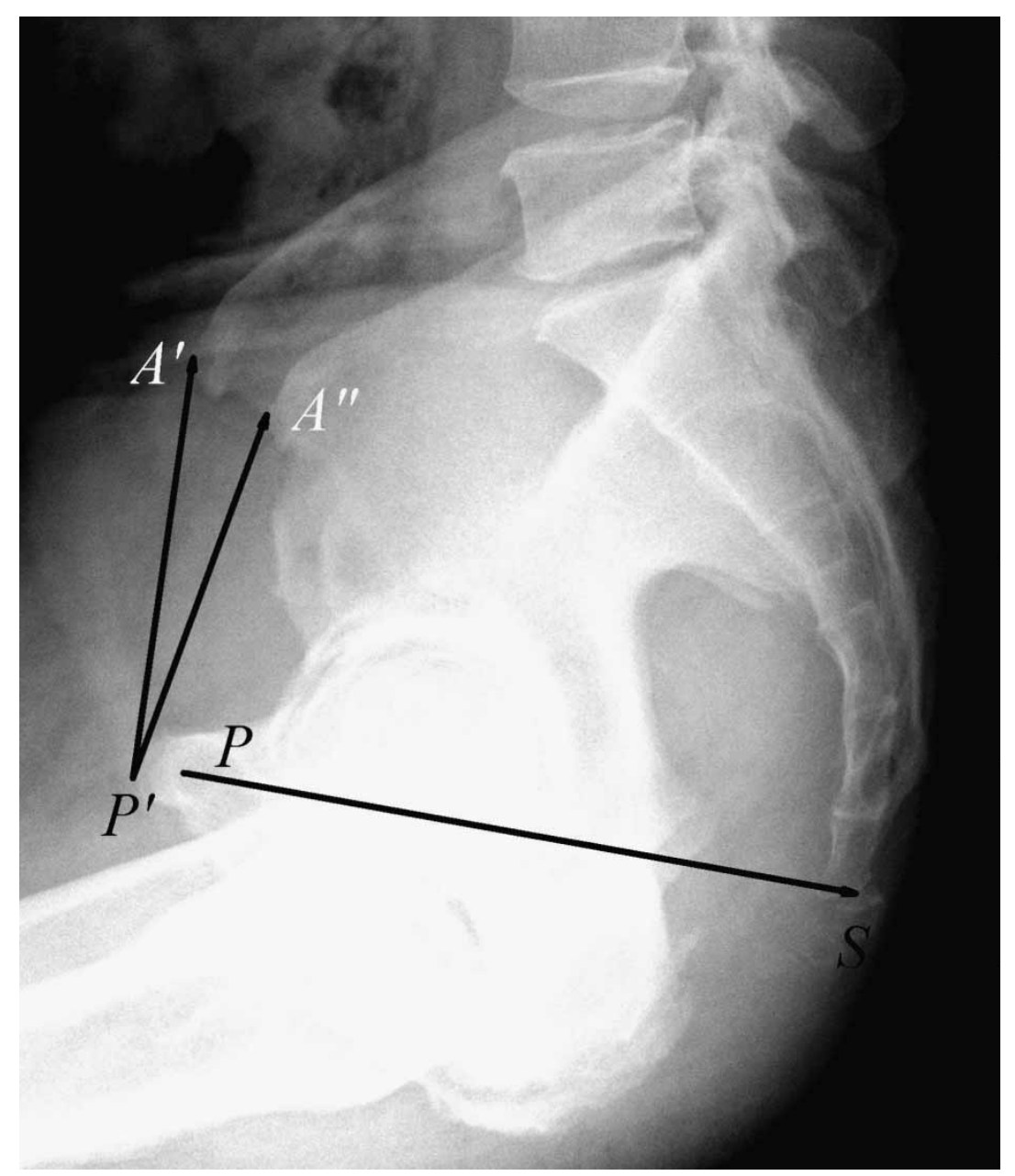

Figure 4. When we measure the length of the pelvis radiographic axis from cross-table lateral view radiograms, we can measure the distance between the centre of the sacrum $(S)$ tip and the centre of the symphis pubis junction $(P)$, that is the length of line $P S$. The angle $\beta$, which is used for correlating with CT standardization, is defined as the angle between the pelvis radiographic axis and anterior pelvis plane. $\beta$ is the average of the angle between line $P S$ and line $P^{\prime} A^{\prime}$ and the angle between line $P S$ and line $P^{\prime} A^{\prime \prime}$. Where $A^{\prime}$ and $A^{\prime \prime}$ mean the two ASIS, $P^{\prime}$ means anterior edge of pubis tubercle. 
oblique-projection-corrected standardization anteversion referenced by

$$
\begin{aligned}
\text { anterior pelvic plane } & =\arcsin \left(\text { vector } a_{\mathrm{sc}} \text { dot vector } n_{\mathrm{sc}}\right) \\
& =\arcsin \left(-\cos \varphi \times \cos \theta_{\mathrm{sc}} \times \cos \beta+\sin \theta_{\mathrm{sc}} \times \sin \beta\right)
\end{aligned}
$$

Traditionally, we can only distinguish anteversion from retroversion by two radiographs, one centred at symphysis pubis (pelvis antero-posterior radiograph) and the other centred at the hip (hip antero-posterior radiograph). If the angle of hip antero-posterior radiograph version is larger than the angle of pelvis antero-posterior radiograph version then the acetabulum is anteversion. This method does not take pelvic flexion into consideration. Our method can extend further. In Equation (A1), we obtain the normal vector of acetabulum however if it is retroversion, the $\theta$ becomes negative. From one radiograph, we cannot distinguish it, however, if we have two radiographs with different orientations and different vertical and horizontal displacements we can compare the two standardized versions. If the difference of these two standardized versions is too large, we can standardize again with an assumption of negative $\theta$. If the difference is much smaller, then retroversion is confirmed, otherwise, it is anteversion. Figures $2 \mathrm{a}$ and $2 \mathrm{~b}$ show the example of distinguishing anteversion from retroversion by two radiographs. By traditional methods, we may mistake it as retroversion, whereas we can confirm it to be anteversion with our method.

We exclude average anteversion smaller than 10 degrees, because we do not want the retroversion acetabulums to cause error when retroversion is mistaken as anteversion, as shown in Figure 2a.

The limitation of this study includes the following items. First of all, this Equation (1) was deduced from the data in 109 subjects with body heights ranging from $142-179 \mathrm{~cm}$, with a mean of $163 \mathrm{~cm}$. Therefore, this equation may not be suitably applied to those with taller or shorter stature. The ssd may need to be measured again for this special situation. We consider that there may be race difference of ssd as well. Before applying our equation for measurement, we recommend to measure a number of patients' ssd in the population to get the normal range and mean value. Finally, we assumed a perfect symmetrical pelvis for these measurements, therefore some errors will be found using this method in the measurement of the asymmetrical pelvis.

\section{Conclusion}

We proposed a new definition of standardized anteversion and developed a mathematical method to measure it. The results showed that the reproducibility of measurement is much better than previous methods. We also provide a mathematical method to convert our standardization anteversion angle to anterior pelvic plane referenced anteversion angle. While the clinical significance of our standardized anteversion is still unclear, future work with this new method could help us clarify the relationship between standardized anteversion and some prognostic factors after total hip arthroplasty.

\section{Acknowledgements}

This study was supported by the grant of NSC96-2320-B-087-001, Taiwan, ROC.

\section{Notes}

1. Tel./Fax: +886-2-27009200. Email: sampliu@ @a.mc.ntu.edu.tw

2. Tel.: +886-2-23123456-3958. Fax: +886-2-23936577. Email: rsyang@ntuh.gov.tw

3. Tel.: +886-2-23562136. Fax: +886-2-23971266. Email: shengmou@ntuh.gov.tw 
4. Tel./Fax: + 886-2-27009200. Email: dienewu@yahoo.com.tw

5. Chen-Kun Liaw and Rong-Sen Yang contributed equally to this work.

\section{References}

[1] M.K. Ackland, W.B. Bourne, and H.K. Uhthoff, Anteversion of the acetabular cup: Measurement of angle after total hip replacement, J. Bone Joint Surg. Br. 68 (1986), pp. 409-413.

[2] American Society for Testing and Materials, Standard practice for conducting an interlaboratory study to determine the precision of a test method, in Annual Book of ASTM Standards. American Society for Test and Materials, Philadelphia, PA, 1999, p. E691.

[3] K. Eckman, M.A. Hafez, B. Jaramaz et al., Accuracy of pelvic flexion measurements from lateral radiographs, Clin. Orthop. 451 (2006), pp. 154-160.

[4] L. Fabeck, D. Farrokh, M. Tolley, P.Y. Descamps, M. Gebhart, and P. Delince, A method to measure acetabulum cup anteversion after total hip replacement, Acta Orthop. Belg. 65 (1999), pp. 485-491.

[5] C.K. Liaw, S.M. Hou, R.S. Yang et al., A new tool for measuring cup orientation in total hip arthroplasties from plain radiographs, Clin. Orthop. 451 (2006), pp. 134-139.

[6] G.E. Lewinnek, J.L. Lewis, R. Tarr, C.L. Compere, and J.R. Zimmerman, Dislocations after total hipreplacement arthroplasties, J. Bone Joint Surg. Am. 60 (1978), pp. 217-220.

[7] R.H. McLaren, Prosthetic hip angulations, Radiology 107 (1973), pp. 705-706.

[8] D.W. Murray, The definition and measurement of acetabular orientation, J. Bone Joint Surg. Br. 75 (1993), pp. $228-232$

[9] H. Olivecrona, L. Weidenhielm, L. Olivecrona, M.O. Beckman et al., A new CT method for measuring cup orientation after total hip arthroplasty: A study of 10 patients, Acta Orthop. Scand. 75 (2004), pp. $252-260$.

[10] R. Pradhan, Planar anteversion of the acetabular cup as determined from plain antero-posterior radiographs, J. Bone Joint Surg. Br. 81 (1999), pp. 431-435.

[11] H. Tannast, U. Langlotz, K.A. Siebenrock, M. Wiese, K. Bernsmann, and F. Langlotz, Anatomic referencing of cup orientation in total hip arthroplasty, Clin. Orthop. 436 (2005), pp. 144-150.

[12] M. Tannast, G. Zheng, C. Anderegg et al., Tilt and rotation correction of acetabular version on pelvic radiographs, Clin. Orthop. 438 (2005), pp. 182-190.

[13] J.D. Visser and J.G. Konings, A new method for measuring angles after total hip arthroplasty: A study of the acetabular cup and femoral component, J. Bone Joint Surg. Br. 63 (1981), pp. 556-559.

[14] K.H. Widmer, A simplified method to determine acetabular cup anteversion from plain radiographs, J. Arthroplasty 19 (2004), pp. 387-390.

\section{Appendix A}

The detailed deduction of our standardization equation is shown below.

When we take an X-ray of the pelvis with the patient in supine position, the X-ray tube is about $100 \mathrm{~cm}$ above the patient, and the patient is lying on the $X$-ray film. At first, we define a 3D Cartesian coordinate system, where the $X-Y$ plane is the $X$-ray film and $X$-axis is parallel to the line passing through both tear drop landmarks of the radiograph and pointed to the right, and $Y$-axis pointed to the patient's head and is perpendicular to $X$-axis. $Z$-axis is defined to point upward and is perpendicular to both $X$-axis and $Y$-axis (Figure 5a).

With this Cartesian coordinate system, we can get the vector of pelvis radiographic axis and normal vector of the acetabulum. Let us assume the radiographic anteversion angle to be $\theta$ and the inclination (abduction) angle to be $\varphi$ of the right hip after total hip arthroplasty. The anteversion $\theta$ and inclination $\varphi$ are measured from this radiograph. According to the definition of trigonometry functions, we can obtain the normal vector of the acetabulum $n$ (Figure 5b).

$$
\text { vector } n=(\sin \varphi \times \cos \theta,-\cos \varphi \times \cos \theta, \sin \theta)
$$

We can also obtain the vector of pelvis radiographic axis by measuring vertical displacement $v \mathrm{~mm}$ and horizontal displacement $h \mathrm{~mm}$. We define vertical displacement $v$ as displacement of sacrococcygeal junction related to the upper pole of symphysis pubis in $Y$-axis direction. We assign $v$ to be positive if sacrococcygeal junction is above the upper pole of symphysis pubis. We define horizontal displacement $h$ as displacement of sacrococcygeal junction relative to the upper pole of symphysis pubis in $X$-axis direction. We assign $h$ to be positive if sacrococcygeal junction is between this acetabulum and the upper pole of symphysis pubis (Figure 2). We assign ssd (mm) as the length 


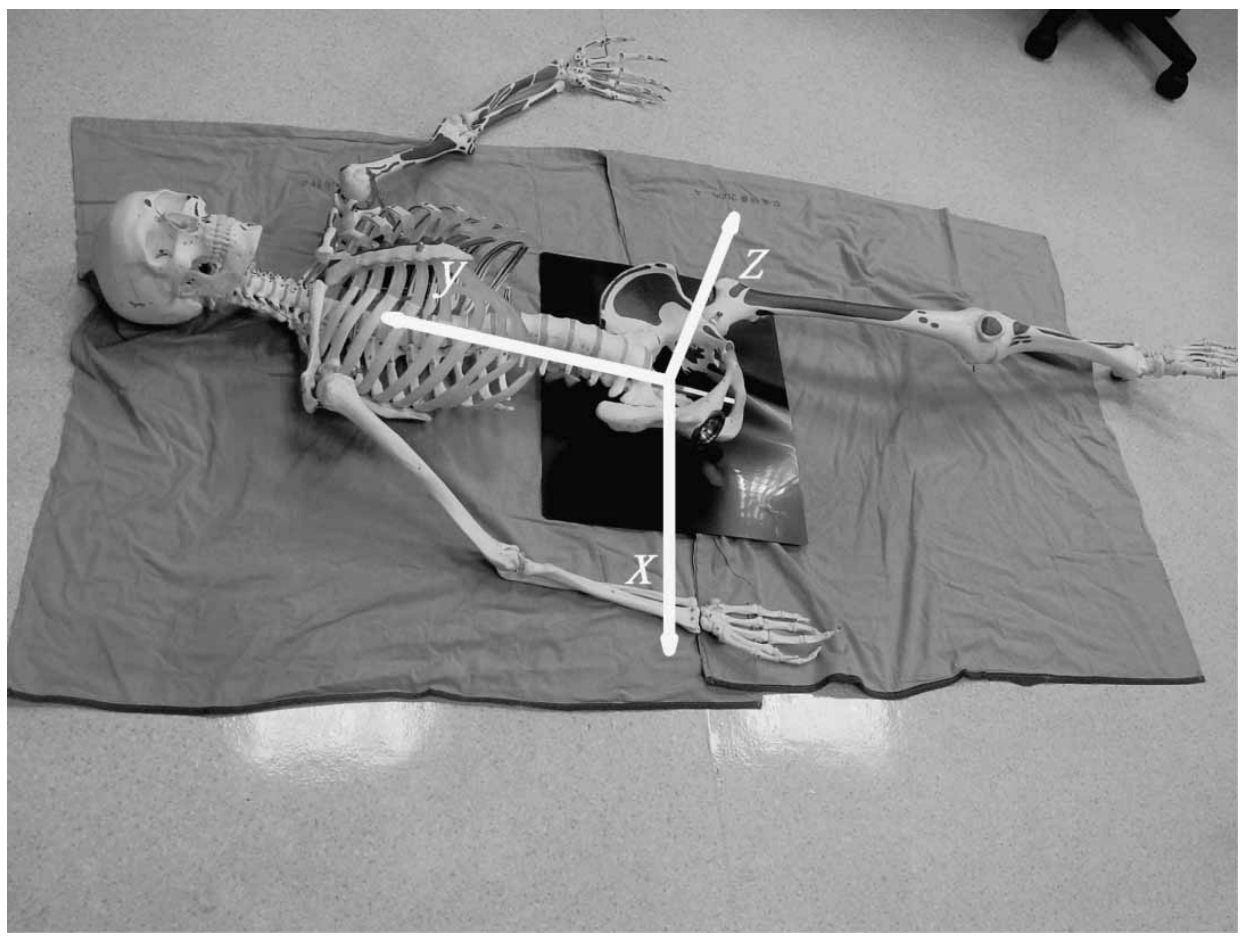

Figure 5a. The 3D Cartesian coordinate system, where $X-Y$ plane is the $\mathrm{X}$-ray film, and $X$-axis is parallel to the line passing through both tear drop landmarks of the radiograph and points to the right, and $Y$-axis points to the patient's head and is perpendicular to $X$-axis. $Z$-axis is defined to point upward and is perpendicular to both $X$-axis and $Y$-axis.

from upper pole of symphysis pubis to the sacrococcygeal junction, we can also obtain vector $a$, vector of pelvis radiographic axis, by the Pythagorean theorem (Figure 5c).

$$
\text { vector } a=\left(-h,-v,\left(\operatorname{ssd}^{2}-h^{2}-v^{2}\right)^{0.5}\right)
$$

According to vector mathematics, we can obtain the angle $\theta$ between two vectors.

$$
\begin{gathered}
\cos \theta=\frac{\text { vector } a \cdot \text { vector } n}{\text { length of vector } a \times \text { length of vector } n} \\
\theta=\arccos \left(\frac{\text { vector } a \cdot \text { vector } n}{\text { length of vector } a \times \text { length of vector } n}\right)
\end{gathered}
$$

where (vector $a \cdot$ vector $n$ ) denotes inner product of vectors $a$ and $n$.

The normal vector is perpendicular to its plane. The radiographic standardized anteversion equals $90^{\circ}$ minus $\theta$.

$$
\text { radiographic standardized anteversion }=90^{\circ}-\theta
$$

$$
\cos \theta=\sin \left(90^{\circ}-\theta\right)
$$

Radiographic standardized anteversion

$$
=\arcsin \left(\frac{-h \times \sin \varphi \times \cos \theta+v \times \cos \varphi \times \cos \theta+\sin \theta \times\left(s s d^{2}-h^{2}-v^{2}\right)^{0.5}}{\operatorname{ssd}}\right)
$$




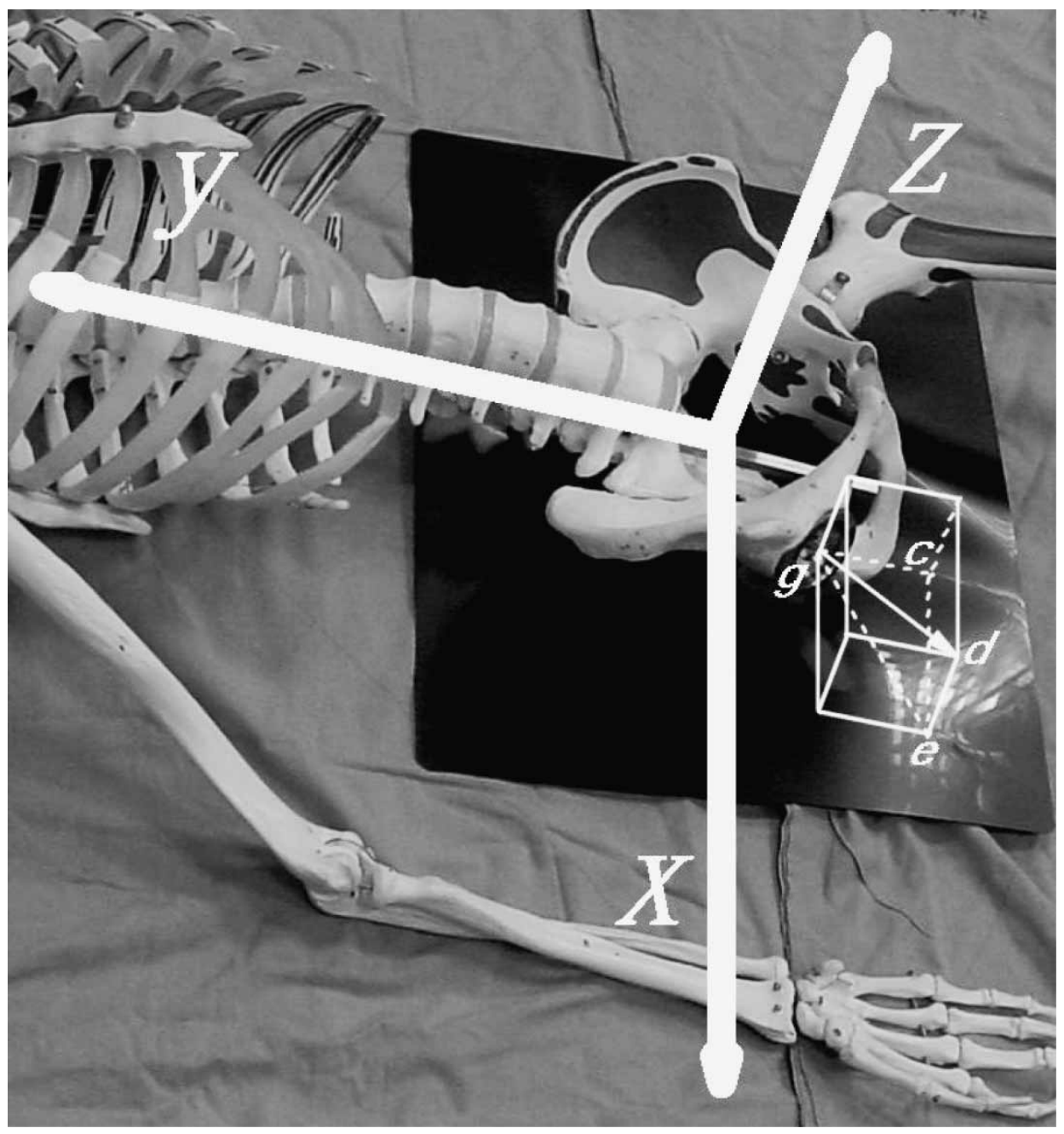

Figure 5b. In the 3D Cartesian coordinate system, the acetabular normal vector $n$ is vector $g d$. The angle $\theta$ formed by line segments $g d$ and $g e$ is the radiographic anteversion. The angle $\varphi$ formed by line segments $g e$ and $g c$ is the inclination. Assume the length of the normal vector $n$ equals $1 \mathrm{~mm}$. According to the definitions of trigonometry functions, the length of line segment ge equals $\cos \theta$ $\mathrm{mm}$; the length of line segment $d e$ equals $\sin \theta \mathrm{mm}$; the length of line segment $g c$ equals $\cos \varphi \times \cos \theta$ $\mathrm{mm}$; the length of line segment $c e$ equals $\sin \varphi \times \cos \theta \mathrm{mm}$. The normal vector $n$ equals $(\sin \varphi$ $\times \cos \theta,-\cos \varphi \times \cos \theta, \sin \theta)$.

We explained how to measure radiographic standardized anteversion of right acetabulum directly. It is similar to measure radiographic standardized anteversion of left acetabulum.

\section{Appendix B}

The detailed deduction of correcting oblique projection of our standardization anteversion angle is shown below.

We define a new 3D Cartesian coordinate system, which is obtained by rotating the aforementioned $Z$-axis to point upward and parallel to the pelvis radiographic axis. 


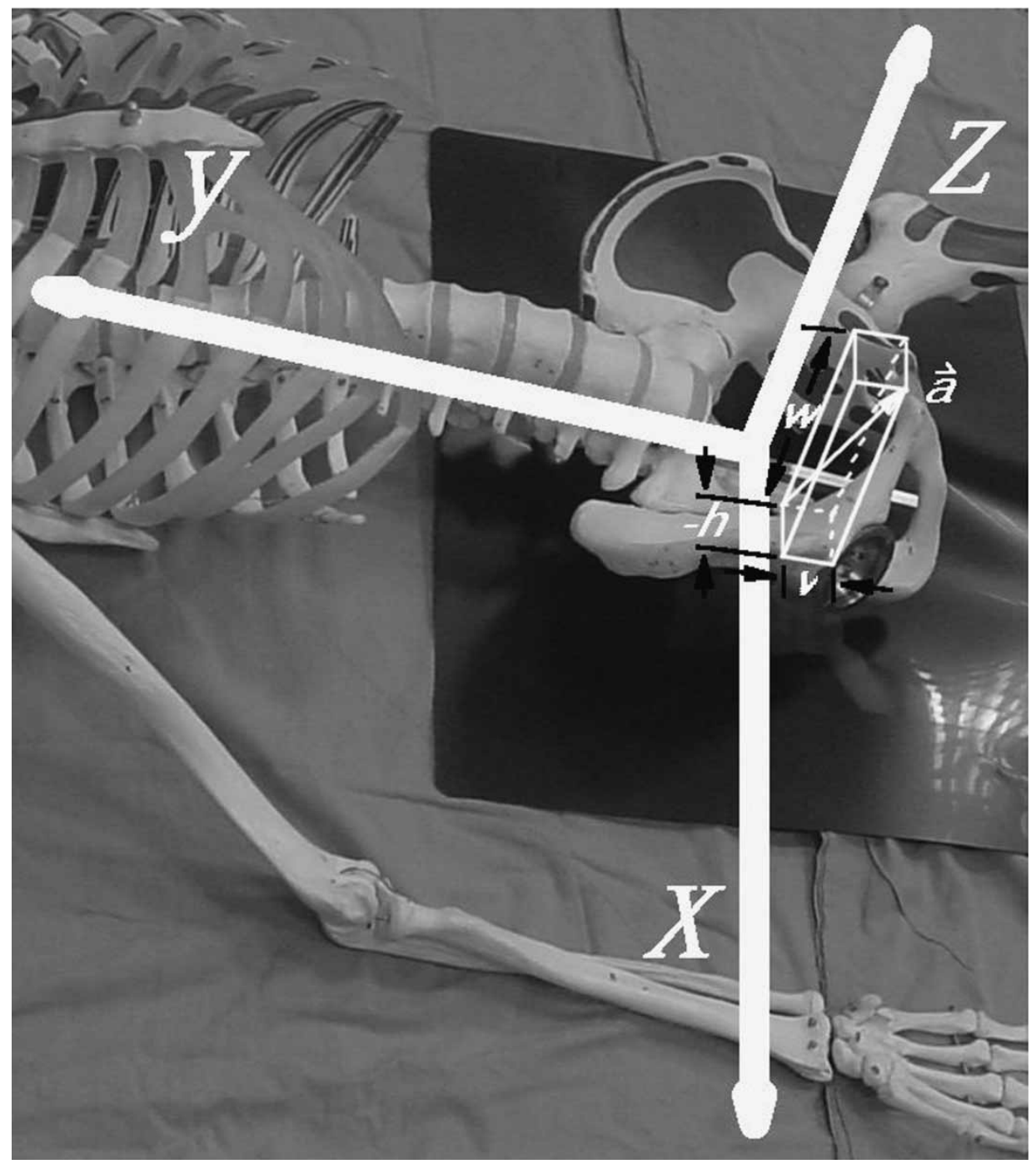

Figure 5c. In the 3D Cartesian coordinate system, the vector of pelvis radiographic axis $a$ is also shown. The horizontal displacement $h$ and vertical displacement $v$ are shown. Because the sacrococcygeal junction is not between this acetabulum and the upper pole of symphysis pubis, the $h$ is negative. The length of the vector $a$ equals ssd (mm). According to Pythagorean theorem, $w$ equals $\left(\operatorname{ssd}^{2}-h^{2}-v^{2}\right)^{0.5}$. Vector $a$ equals $\left(-h,-v,\left(\operatorname{ssd}^{2}-h^{2}-v^{2}\right)^{0.5}\right)$.

With this new Cartesian coordinate system, we can obtain the acetabulum's normal vector with Equation (A1), where the anteversion becomes radiographic standardized anteversion $\theta_{\mathrm{s}}$. The inclination $\varphi$ is unchanged because Tannast et al. had proved it to be almost unchanged [10].

$$
\text { vector } n_{\mathrm{s}}=\left(\sin \varphi \times \cos \theta_{\mathrm{s}},-\cos \varphi \times \cos \theta_{\mathrm{s}}, \sin \theta_{\mathrm{s}}\right)
$$

We define a new vector $r$, originated from centre of the acetabulum to the source of the X-ray.

$$
\text { vector } r=(-d, 0,100)
$$


where $d$ means the distance from symphysis pubis to the acetabulum centre measured from radiographs.

The oblique-projection-corrected anteversion means the angle between vector $r$ and acetabulum plane.

$$
\text { vector } a_{\mathrm{s}} \cdot \text { vector } r=100 \times \sin \theta_{\mathrm{s}}-d \times \sin \varphi \times \cos \theta_{\mathrm{s}}
$$

According to the same principles of Equations (A3)-(A6) and A, we obtain the obliqueprojection-corrected anteversion:

$$
\text { oblique-projection-correctedanteversion }=\theta_{\mathrm{sc}} \operatorname{arc} \sin \left(\frac{100 \times \sin \theta_{s}-d \times \sin \varphi \times \cos \theta_{s}}{100^{2}+d^{2}}\right)^{0.5}
$$

\section{Appendix C}

The detailed deduction of converting our oblique-projection-corrected standardization anteversion angle to anterior pelvic plane referenced anteversion angle is shown below.

The angle $\beta$ is defined as the angle between pelvis radiographic axis and the anterior pelvis plane. It is measured from cross-table lateral view of the pelvis. Because we assume the pelvis to be perfectly symmetrical, it is measured from cross-table lateral view including pubis tubercles, ASIS and the sacrococcygeal junction (Figure 4). We can obtain the normal vector of anterior pelvis plane by rotating the pelvis radiographic axis upward $(90-\beta)$ degrees.

We define a new 3D Cartesian coordinate system, which is obtained by rotating the previous $Z$-axis to point upward and parallel to the pelvis radiographic axis.

With this new Cartesian coordinate system, we can obtain the acetabulum's normal vector with Equation (A1), where the anteversion becomes oblique-projection-corrected radiographic standardized anteversion $\theta_{\mathrm{sc}}$. The inclination $\varphi$ is unchanged because Tannast et al. [10] had proved it to be almost unchanged.

$$
\text { vector } n_{\mathrm{sc}}=\left(\sin \varphi \times \cos \theta_{\mathrm{sc}},-\cos \varphi \times \cos \theta_{\mathrm{sc}}, \sin \theta_{\mathrm{sc}}\right)
$$

According to the definition of trigonometric functions, we can obtain the normal vector of anterior pelvis plane.

$$
\begin{aligned}
\text { vector } a_{\mathrm{sc}} & =\left(0, \sin \left(90^{\circ}-\beta\right), \cos \left(90^{\circ}-\beta\right)\right) \\
\text { vector } a_{\mathrm{sc}} \text { vector } n_{\mathrm{sc}} & =-\cos \varphi \times \cos \theta_{\mathrm{sc}} \times \cos \beta+\sin \theta_{\mathrm{sc}} \times \sin \beta
\end{aligned}
$$

Because both vectors are unit vectors, we can obtain the anteversion by vector mathematics.

oblique-projection-corrected standardization anteversion referenced by anterior pelvic plane

$$
=\arcsin \left(-\cos \varphi \times \cos \theta_{\mathrm{sc}} \times \cos \beta+\sin \theta_{\mathrm{sc}} \times \sin \beta\right)
$$




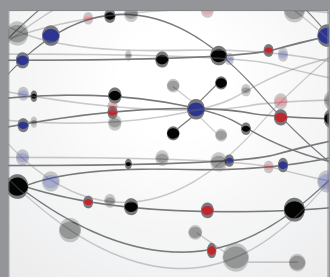

The Scientific World Journal
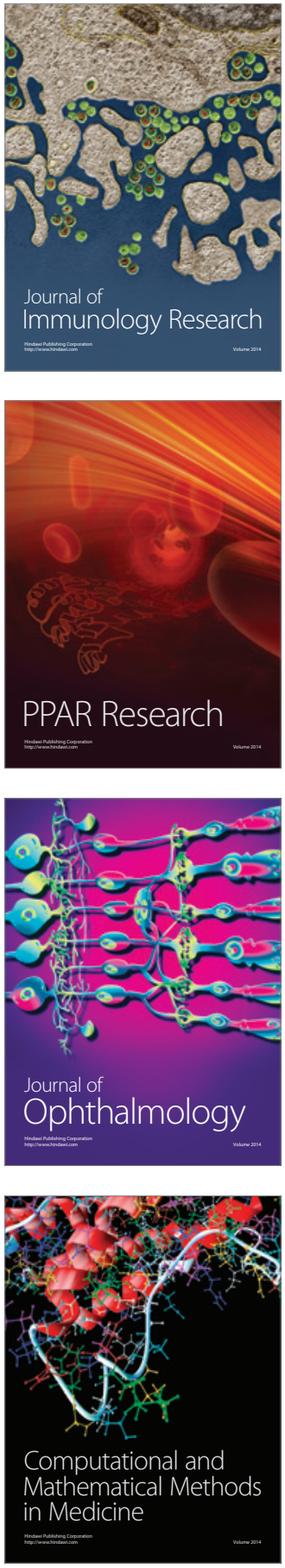

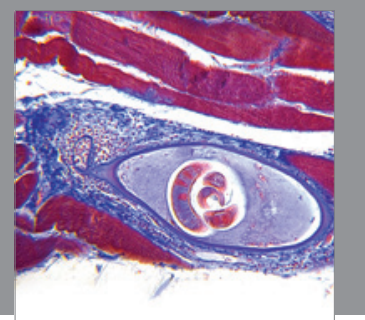

Gastroenterology

Research and Practice
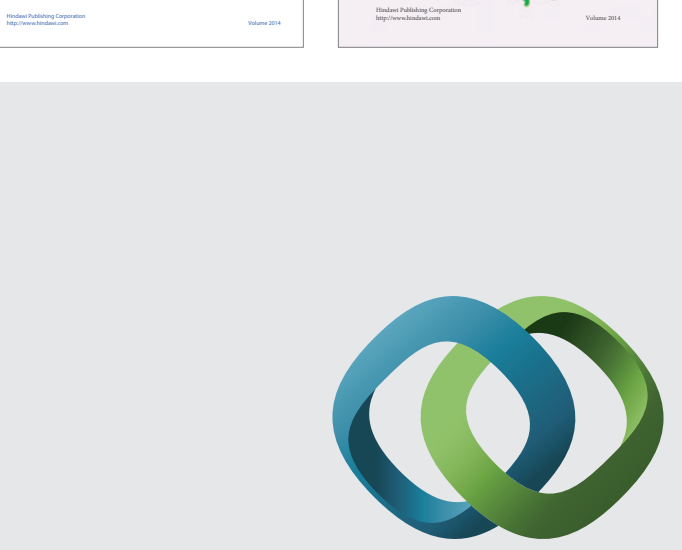

\section{Hindawi}

Submit your manuscripts at

http://www.hindawi.com
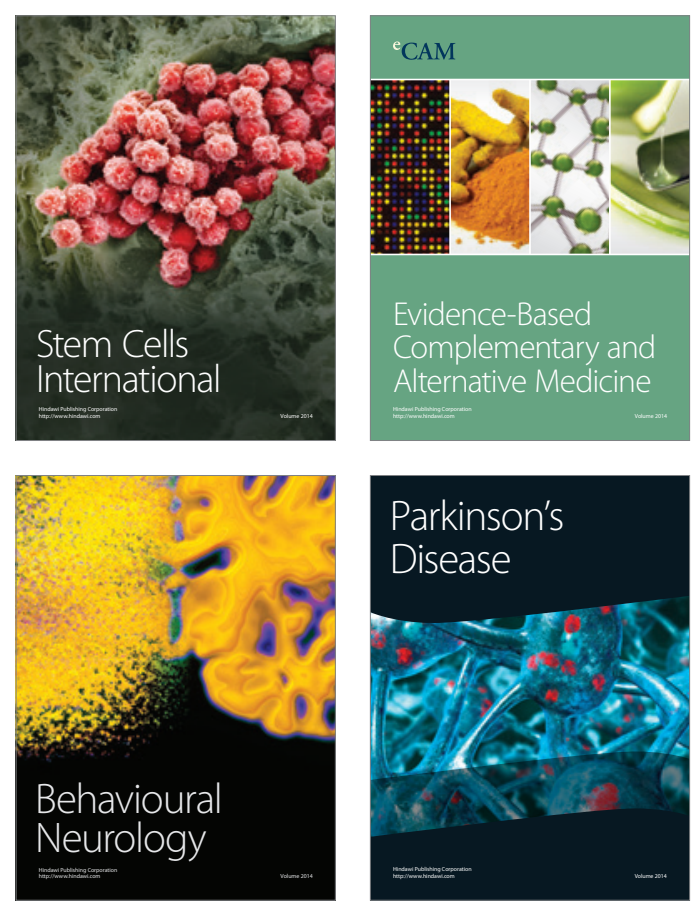

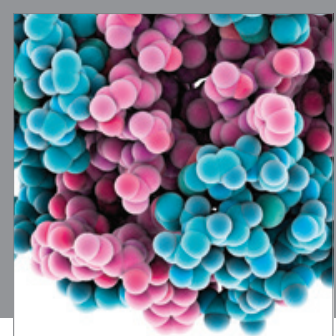

Journal of
Diabetes Research

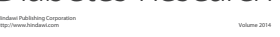

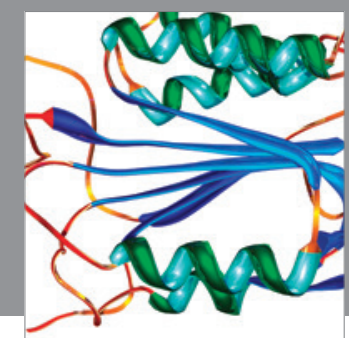

Disease Markers
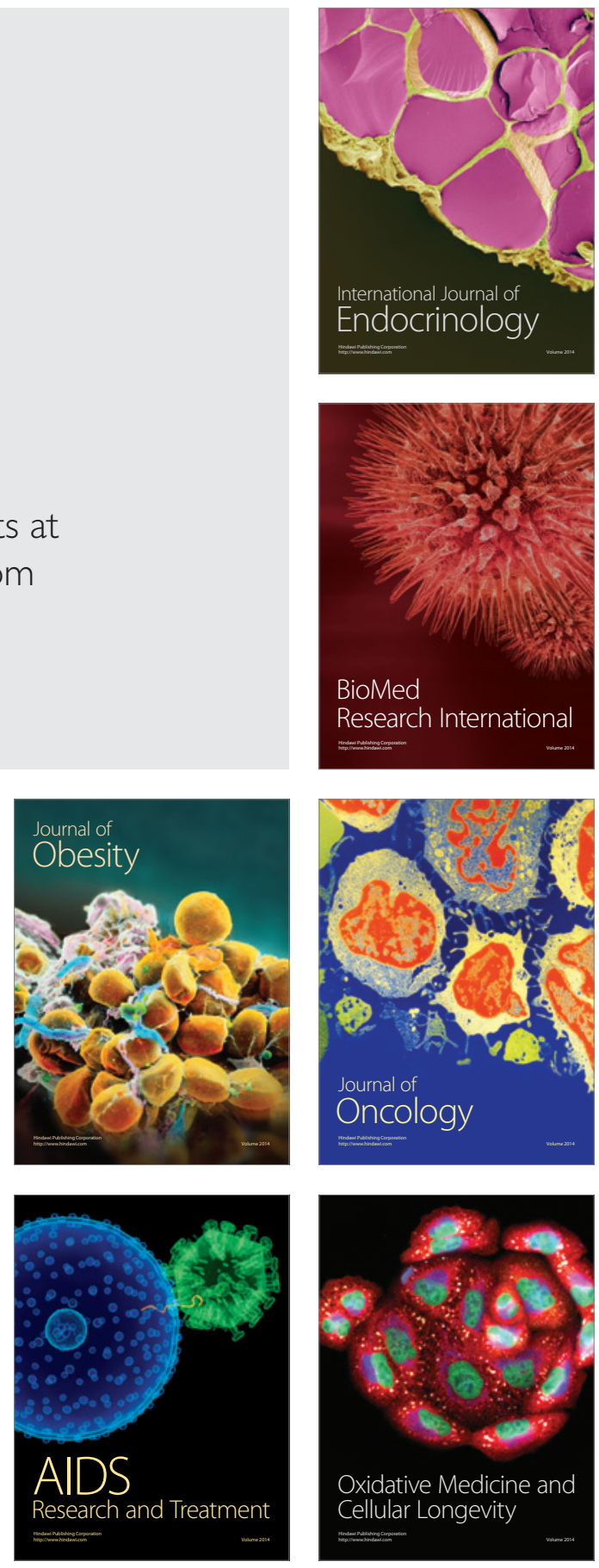